Study of hepatitis E virus ORF2 antigen kinetics in human-liver chimeric mice and its impact on

\title{
HEV diagnosis
}

Ibrahim M. Sayed ${ }^{1,2, a}$, Lieven Verhoye ${ }^{1}$, Claire Montpellier ${ }^{3}$, Florence Abravanel ${ }^{4,5,6}$, Jacques Izopet $^{4,5,6}$, Laurence Cocquerel ${ }^{3}$, Philip Meuleman ${ }^{1^{*}}$

${ }^{1}$ Laboratory of Liver Infectious Diseases, Faculty of Medicine and Health Sciences, Ghent University, Ghent, Belgium.

${ }^{2}$ Microbiology and Immunology Department, Faculty of Medicine, Assiut University, Assiut, Egypt ${ }^{3}$ University of Lille, CNRS, INSERM, CHU Lille, Pasteur Institute of Lille, U1019-UMR 8204-CIIL- Center for Infection and Immunity of Lille, F-59000 Lille, France INSERM U1043, IFR-BMT, CHU Purpan, Toulouse, France

${ }^{5}$ Université Paul-Sabatier, Toulouse, France

${ }^{6}$ Laboratory of Virology, CHU Purpan, Toulouse, France

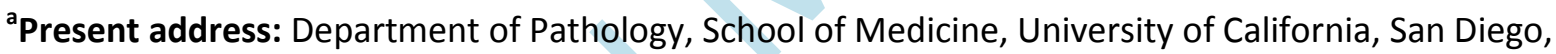
La Jolla, California, USA.

*Corresponding author:

Prof. Dr. Philip Meuleman

Laboratory of Liver Infectious Diseases

Dept. of Diagnostic Sciences

Faculty of Medicine and Health Sciences - Ghent University

Phone: +3293323658

E-mail: philip.meuleman@ugent.be

Word count in text: 3500 words

(C) The Author(s) 2019. Published by Oxford University Press for the Infectious Diseases Society of America. All rights reserved. For permissions, e-mail: journals.permissions@oup.com. 


\section{Abstract}

Background: Hepatitis E virus infection (HEV) is an emerging problem in developed countries.

Diagnosis of HEV infection is based on the detection of HEV-specific antibodies, viral RNA and/or antigens (Ag). Humanized mice were previously reported as a model for the study of HEV infection, but published data was focused on the quantification of viral RNA. However, the kinetics of HEV Ag expression during the course of infection remains poorly understood.

Methods: Plasma and fecal suspensions from HEV infected and ribavirin-treated humanized mice were analyzed using HEV antigen ELISA, RT-qPCR, density gradient and Western blotting.

Result: ORF2 Ag was detected in both plasma and stool of HEV infected mice, and increased overtime. Contrary to HEV RNA, ORF2 Ag levels were higher in mouse plasma than in stool. Interestingly, ORF2 was detected in plasma of mice that were RNA negative in plasma but RNA positive in stool; and after viral clearance by ribavirin. Plasma density gradient analysis revealed the presence of the non-infectious glycosylated form of ORF2.

Conclusion: ORF2 Ag can be used as a marker of active HEV infection and the assessment of antiviral therapy, especially when fecal samples are not available or molecular diagnostic tests are not accessible.

Key words: HEV Ag, humanized mice, ORF2, diagnosis, ribavirin therapy 


\section{Introduction}

Hepatitis E virus (HEV) causes about 20 million infection annually[1]. HEV is a positive sense single stranded RNA virus that belongs to the Hepeviridae family, comprising Orthohepevirus and Pescihepevirus genera. The Orthohepevirus A species includes at least 7 genotypes (gt), 5 of which were shown to be capable of causing infection in humans[2, 3]. HEV gt1 and gt2 isolates infect humans only and are dominant in developing countries, while gt 3 and gt4 isolates are zoonotic and are more common in industrialized countries[4]. HEV isolates of gt5 and gt6 have been isolated from wild boar in Japan and seem not infectious to humans[5]. HEV gt7 was isolated from camels in the Middle East[6]. To our knowledge, only one human case was diagnosed with camelid HEV infection[7]. More recently, a potential $8^{\text {th }}$ genotype was identified in Bactrian camels in China[8]. There are four major routes of HEV transmission: fecal-oral, food borne, blood transfusion and vertical transmission from infected mothers to their babies[1, 4]. Although a typical HEV infection will resolve spontaneously, chronicity has been observed in immunocompromised patients[9]. Ribavirin (RBV) is the drug of choice for treatment of chronic HEV infection[10, 11].

The HEV genome encodes three open reading frames (ORFs); ORF1, ORF2 and ORF3. ORF1 encodes a non-structural polyprotein that is essential for viral replication. ORF2 encodes the viral capsid protein and is the most immunogenic viral protein. ORF3 encodes a small phosphoprotein that is involved in virion morphogenesis and egress $[1,4]$.

The diagnosis of HEV infection is based mainly on the detection of HEV RNA (gold standard) and/or detection of anti-HEV antibodies (IgM \& IgG)[12]. Recently, a novel diagnostic assay became commercially available (Bejing Wantai Biological Pharmaceutical Co., Bejing, China), which is based on the detection of HEV ORF2 antigen (Ag). Previous studies showed that this HEV Ag ELISA could be used as a diagnostic tool in clinical laboratories where molecular assays are lacking[13-16]. Behrendt et al. showed that the sensitivity of the HEV Ag ELISA assay is less than RT-qPCR, especially during acute HEV infection, and that higher HEV Ag levels were detected in chronically infected patients 
compared to acute ones[17]. They also reported that HEV Ag was detectable for more than 100 days after HEV RNA clearance in ribavirin treated patient[17]. Moreover, Marion et al., reported that the serum level of HEV Ag at the acute phase of HEV infection in immunocompromised patients could predict the possibility of HEV chronicity in these patient [18]. Using an HEV cell culture system, Montpellier et al., showed that HEV produces 3 different forms of ORF2: infectious/intracellular ORF2 (ORF2i), glycosylated ORF2 (ORF2g) and cleaved ORF2 (ORF2c). The ORF2i protein(80KDa) is the structural component of infectious viral particles and is not glycosylated. In contrast, ORF2g (90KDa) and ORF2c (75KDa) proteins are glycosylated forms that are secreted in large amounts in culture supernatant and are not associated with infectious virions. ORF2g and ORF2c do not form particulate material but, importantly, are the most abundant antigens in patient sera[19]. Recently, Yin et al. reported that the glycosylated secreted form of ORF2 resembles the virus capsid, but lacks the antigenic epitope predicated to bind the cell receptor[20].

Recently, our group and others have established human-liver chimeric mice as a small animal model for the study of HEV infection and the evaluation of novel antiviral therapies [21-26]. Similar to immunocompromised patients, these immunodeficient mice (SCID or Rag- $2^{-1-}-\mathrm{IL}-2 \mathrm{R} \gamma^{-/-}$) develop chronic HEV infection when challenged with different HEV preparations of gt1 and gt3 [21-24]. While HEV infection was confirmed in these models by the detection and quantification of HEV RNA in mouse stool, plasma, bile and liver tissue, the kinetics and characteristics of HEV antigen expression during the course of infection remained unclear.

In this study, we characterized the ORF2 antigen secretion during the course of HEV infection in humanized mice and investigated its potential use as marker for antiviral therapy. 


\section{Material and Methods}

\section{1-Production, infection and therapy of human liver chimeric mice}

$\mathrm{UPA}^{+/+}-\mathrm{SCID}$ and FRG mice were transplanted with primary human hepatocytes as previously described[21, 23, 27]. Humanized mice were inoculated intrasplenically or orally with fecal suspensions or plasma containing HEV of gt1 or gt3. Plasma and fecal samples were regularly collected from the inoculated mice. Ribavirin (RBV) treatment was performed at $50 \mathrm{mg} / \mathrm{kg}$ as previously described [21]. Samples used in this study originated both from mice used in previous studies $[21,23,25]$ and new infections. Pharmacokinetic analysis of HEV ORF2 antigen in nonhumanized mice are described in the supplemental materials and methods section. All procedures were performed according to European and Belgian legislation; and were approved by the Animal Ethics Committee of the Faculty of Medicine and Health Sciences of Ghent University.

\section{2-Detection and quantification of HEV RNA in mouse samples}

Viral RNA was extracted from $10 \%(\mathrm{w} / \mathrm{v})$ mouse fecal preparations and mouse plasma, HEV RNA was detected and quantified as described previously[21].

\section{3-Detection of HEV Ag in mouse samples}

Detection of HEV Ag in mouse samples was performed using the HEV-Ag ELISA ${ }^{\text {Plus }}$ assay (Bejing Wantai Biological Pharmaceutical Co., China) according to the manufacturer's instructions, with slight modifications to determine the cut-off (C.O.), as described in the supplementary material and methods section.

\section{4-Density gradient analysis}

Mouse plasma and $10 \%(\mathrm{w} / \mathrm{v})$ mouse stool suspensions were prepared from HEV-infected and noninfected mice, and were ultra-centrifuged as previously described[21]. More details are available in the supplementary material and methods section 


\section{5- Statistic analyses}

The geometric mean of the viral load and HEV Ag level was determined in mouse samples at the start of RBV therapy and EOT. Statistical significance was calculated by GraphPad Prism version 6.1 using a paired two-tailed Student's t-test. 


\section{Results}

\section{1- Kinetic analysis of HEV Ag secretion in HEV-infected human-liver chimeric mice}

Plasma and $10 \%(\mathrm{w} / \mathrm{v})$ fecal suspensions from HEV infected humanized mice were analyzed for HEV RNA and ORF2 Ag. The HEV RNA data was extensively described in our previous publications[21, 23], but we here correlate the viral RNA levels with the amount of ORF2 antigen present in each sample. In parallel to the HEV RNA load, the level of HEV Ag increased over time in both plasma and $10 \% \mathrm{w} / \mathrm{v}$ fecal preparations of HEV gt1 (Figure 1) and gt3 (Figure 2) infected mice. Similar to the HEV RNA load, the observed HEV Ag levels were considerably higher in HEV gt1-infected mice compared to those in gt3-infected mice (Figure 1 and 2).

When comparing the ratio of HEV RNA to HEV ORF2 $\mathrm{Ag}$, we observed an overall inverse relation between the plasma and the feces. Plasma contained relatively higher levels of ORF2 Ag than HEV RNA, while at the corresponding time points the relative level of viral RNA in fecal suspensions was higher than the amount of ORF2 Ag (Figure 1). This indicates that the large amounts of ORF2 Ag present in mouse plasma likely correspond to non-infectious ORF2 Ag, as previously shown for human sera [19].

HEV ORF2 Ag levels were very low (near or below cut-off) in mouse stool samples, especially during the early phase of infection where the viral load was already relatively high in most animals (RNA level up to $10^{5}-10^{6} \mathrm{IU} / \mathrm{ml}$ )(Figure 1 and 2). In one mouse, the viral RNA was detectable in stool by RTqPCR (Figure 2B) starting from week 2 until week 16 post inoculation. However, HEV ORF2 levels remained below cut-off in all tested fecal samples. On the other hand, HEV ORF2 Ag was detected in certain mouse plasma samples in which HEV RNA was under limit of detection (Figure 2A and C), confirming our hypothesis that the non-infectious forms of ORF2 were abundantly present in mouse plasma. 
As can be seen in Figure 1 and 2, ORF2 levels increased over time, especially in HEV gt1-infected mice, but this increase was not always concomitant with an increase in HEV RNA load. In one mouse (Figure $1 \mathrm{C}$ ), viremia was nearly stable during the course of infection, but the ORF2 Ag level increased sharply over the same 5-week period. This again suggests that the non-infectious forms of ORF2 accumulate in the plasma of infected mice.

\section{2- ORF2 status in humanized mice with low HEV replication and in mice after oral HEV challenge.}

Next, we examined the HEV ORF2 Ag status in samples obtained from mice with low HEV replication. In these mice, the viral load was under limit of quantification (LOQ) in the plasma during the course of HEV infection, while HEV RNA was continuously detectable in feces but at a relatively low level $\left(2 \times 10^{3}\right.$ to $\left.5 \times 10^{4} \mathrm{IU} / \mathrm{ml}\right)$. As shown in Figure 3, HEV ORF2 was not detected in any of the tested plasma and stool samples.

Similarly, we evaluated whether HEV ORF2 could be detected in humanized mice that were orally inoculated with multiple HEV preparations. We have previously shown that oral inoculation does not lead to HEV infection and that HEV RNA remains under LOD in the plasma and stool[21, 23]. Here, we detected ORF2 only the first week after inoculation, and only in stool suspensions (Supplemental Figure 1). HEV ORF2 was never detected in any of the subsequent samples.

\section{3- Characterization of HEV ORF2 present in mouse samples}

lodixanol density gradient centrifugation was performed on mouse plasma and $10 \%(\mathrm{w} / \mathrm{v})$ mouse stool suspensions collected at different time points after viral inoculation to evaluate which of the different ORF2 forms were present. RT-qPCR, HEV ORF2 Ag ELISA and WB analysis were performed on each fraction. We first focused on the analysis of mouse plasma samples collected before and 1,5 and 10 weeks after infection. As shown in Figure 4, the peak of HEV RNA was identified in fraction 6 (density $1.11 \mathrm{~g} / \mathrm{l}$ ), while the peak of HEV Ag was always observed in fraction 4(density $1.09 \mathrm{~g} / \mathrm{l}$ ). One week after viral inoculation, HEV ORF2 was detected by ELISA in fraction 4 and not in the RNA 
enriched infectious fraction 6 . Western blot analysis confirmed the observations made by ELISA and revealed that the ORF2g protein (90 kDa non-infectious glycosylated form) was the only form detectable in plasma.

At later time points (week 5 and 10 post-infection), HEV ORF2 Ag became detectable in more density gradient fractions, confirming our previous observation that the secretion of HEV ORF2 Ag is a slow and accumulative process. HEV ORF2 was detected in different mouse plasma fractions which did not contain HEV RNA. Western blot analysis revealed that the ORF2g protein remained the major ORF2 form in mouse plasma at these later time points (Figure 4). We did not detect the infectious ORF2form (ORF2i) in any of the mouse plasma fractions at any time point.

Density gradient analysis of fecal samples revealed that the peak of HEV RNA and HEV ORF2 Ag was found in fraction $10(1.16 \mathrm{~g} / \mathrm{l})$ and fraction $3(1.07 \mathrm{~g} / \mathrm{l})$, respectively (Supplementary Figure $2 \mathrm{~A})$. The relative amount of HEV ORF2 Ag was lower in stool fractions compared to plasma fractions, while this was the opposite for the HEV RNA level. Again, HEV ORF2 Ag was not detected by ELISA in the stool infectious fraction (fraction 10). In addition, we did not visualize any form of ORF2 in the stool fractions using Western blotting (data not shown). Unlike the HEV RNA distribution, the distribution of HEV ORF2 Ag among the different stool fractions was similar to the distribution of ORF2 Ag among plasma fractions (Supplementary Figure $2 \mathrm{~B}$ and $\mathrm{C}$ ).

\section{4-Kinetics of HEV ORF2 Ag in humanized mice during the course of ribavirin therapy}

Five HEV infected humanized mice underwent a 2-week ribavirin (RBV) therapy at $50 \mathrm{mg} / \mathrm{kg}$. HEV ORF2 Ag and HEV RNA were tested both in plasma and $10 \%(\mathrm{w} / \mathrm{v})$ stool suspensions at 3 different moments: start of therapy (SOT), end of therapy (EOT) and at viral relapse. Similar to the effect on viral RNA load, RBV therapy caused a reduction in the level of ORF2 in both the stool and the plasma of the treated mice (Figure 5). Although, the reduction in viremia was more pronounced than the reduction in fecal RNA load, the reduction of ORF2 in plasma was less than the reduction in fecal ORF2Ag. At the SOT, the geometric mean of fecal RNA load and HEV Ag level in five mice was $5.1 \times 10^{6}$ 
$\mathrm{IU} / \mathrm{ml}$ and $12.48 \mathrm{~A} /$ C.O., respectively, and were reduced at EOT to $2.05 \times 10^{5} \mathrm{IU} / \mathrm{ml}$ and $3.75 \mathrm{~A} / \mathrm{C} . \mathrm{O}$., respectively (Figure $5 \mathrm{~A}$ and $\mathrm{B}$ ). While the geometric mean of viremia and plasma $\mathrm{HEV} \mathrm{Ag} \mathrm{level} \mathrm{in}$ these mice at the start of therapy were $2.95 \times 10^{4} \mathrm{IU} / \mathrm{ml}$ and $26.1 \mathrm{~A} / \mathrm{C} . \mathrm{O}$., respectively, and their levels were reduced to the limit of quantification $\left(\mathrm{LOQ}=4.05 \times 10^{2} \mathrm{IU} / \mathrm{ml}\right)$ and $14.49 \mathrm{~A} / \mathrm{C}$.O., respectively at EOT (Figure 5A and B).The reduction in the fecal HEV Ag, but not plasma HEV Ag, was statistically significant ( $P=0.0008)$ (Figure 5A). Interestingly, the level of HEV Ag was increased again in both the plasma and stool of the treated mice after cessation of therapy (Figure 5C).

\section{5- Assessment of in vivo stability of HEV ORF2}

In order to evaluate how long ORF2 remains detectable in plasma and feces after secretion from the liver, we inoculated two groups of non-humanized mice with iodixanol cushion-isolated HEV ORF2 preparations containing either the three forms of ORF2 (ORF2i, ORF2g and ORF2c), or the noninfectious form of ORF2 (ORF2g and ORF2c). The amount of HEV RNA and ORF2 protein present in plasma and feces were quantified daily. HEV ORF2 Ag was detectable in mouse stool and plasma of both groups, but the level decreased gradually until ORF2 disappeared completely within 1 week (Supplementary Fig 3). HEV RNA was under LOQ in all tested samples. 


\section{Discussion}

$\mathrm{HEV}$ virus infection is mainly diagnosed by detection of HEV-specific IgM antibodies and/or detection of HEV RNA. A diagnostic ELISA assay based on the detection of HEV ORF2 Ag in patient plasma samples became recently commercially available. The sensitivity and specificity of this assay was reported previously[13-15, 17], and it is recommended in clinical settings where molecular diagnosis is not available. HEV Ag can be used as a diagnostic marker in the window period and in chronic HEV infection, especially in immunocompromised patients in whom seroconversion may be delayed or absent $[15,17,28,29]$.The kinetics of HEV Ag detection during the course of HEV infection is not known. Here, we used human liver chimeric mice (UPA-SCID and FRG background) to study the kinetics of HEV Ag expression during the course of HEV infection and therapy. Since the adaptive immune system is lacking in these mice, chronic HEV infection is developed when these mice are challenged with HEV preparations.

HEV Ag was detected in both mouse plasma and stool preparations of HEV infected humanized mice. In addition, the Ag level increased with time suggesting that HEV Ag is a relevant marker of active HEV replication. The level of HEV Ag was higher in mouse samples at later time points of infection, indicating that the production of HEV Ag is cumulative. In a similar manner, Behrendt et al.reported that the HEV Ag level can differentiate between acute and chronic HEV infection; i.e. higher HEV Ag levels were detected in chronic HEV infected patients [17]. HEV Ag levels were higher in HEV gt1 infected mice than in HEV gt3 infected mice, confirming our previous data showing that HEV gt3 is less virulent than HEVgt1[21]. In contrast to the HEV RNA load, HEV Ag was relatively higher in mouse plasma than in fecal suspensions. In addition, HEV Ag was detected in a few plasma samples that scored negative for HEV RNA, suggesting that detected Ag likely corresponds to non-infectious ORF2 proteins. Our results are in agreement with previous studies that proposed that non-infectious ORF2 proteins are the major antigens in cell culture supernatant and patient sera[19]. Previous studies showed that the ratio of $\mathrm{HEV} \mathrm{Ag/RNA} \mathrm{was} \mathrm{significantly} \mathrm{higher} \mathrm{in} \mathrm{the} \mathrm{urine} \mathrm{compared} \mathrm{to} \mathrm{the}$ 
serum of the same patient $[18,30]$. In humanized mice, the liver is the main site of infection and previously we could not detect HEV RNA in the kidney nor the brain of the infected mice [25].

HEV Ag was not be detected in HEV infected mice which were non-viremic and in which the fecal viral load was relatively low. This is probably due the limited amount of Ag secreted into the plasma that was too low to be detectable by ELISA. Our results suggest that in case low-level HEV replication is expected, qPCR analysis on stool samples is the best option for diagnosis, especially in immunocompromised patients where seroconversion is delayed. Similarly, several groups reported that HEV-Ag ELISA assay is less sensitive than PCR especially when the viral load is low $[12,14,15$, 17]. In addition, HEV Ag was not detected in mice that were inoculated orally with HEV preparations, thereby confirming our previous observation that the oral route is not a suitable way to establish HEV infection in humanized mice $[21,23]$, probably due to lack and/or different essential receptors in the murine (non-humanized) gut.

Analysis of mouse gradient fractions showed that the peak of HEV Ag is different from the peak of HEV RNA. The peak of HEV RNA in mouse stool sample was $1.16 \mathrm{~g} / \mathrm{l}$ and the peak of HEV RNA in mouse plasma was $1.11 \mathrm{~g} / \mathrm{l}$. This difference may be attributed to the presence of lipids around or associated with the virions that circulate in the plasma [21]. On the other hand, the distribution of HEV Ag in plasma was similar to that in stool. The ORF2g protein was the major ORF2 form detected in mouse plasma. Our results agree with Montpellier et al., who showed that the peak of HEV Ag in patient plasma samples was at $1.08 \mathrm{~g} / \mathrm{l}$, and non-infectious ORF2 proteins (ORF2c/ORF2g) were the major Ag present[19]. HEV Ag could be detected in some mouse plasma fractions that were devoid of viral nucleic acid. Similarly, Behrendt et al. detected HEV Ag in all gradient fractions of HEV patient sera suggesting the presence of distinct fragments of the viral capsid protein with different densities[17]. The presence of high levels of non-infectious ORF2 in mouse plasma might also explain the low infectivity of plasma preparation compared to stool preparation [21, 23].The amount of HEV Ag present in mouse stool fraction was relatively low, which impeded its characterization by WB. 
Next, we tested the effect of RBV therapy on the HEV Ag level in mouse samples. We found that RBV causes a reduction in both the plasma and fecal HEV Ag level, and the reduction was statistically significant in mouse stool samples but not in plasma. In all treated mice, the reduction of HEV Ag was concomitant with the reduction of HEV RNA in mouse stool, while most mice became nonviremic at EOT, HEV Ag remained detectable in mouse plasma. This indicates that viral Ag remains present in mouse plasma even after clearance of infection. Our results agree with Behrendt et al., who reported that HEV Ag could be detected in patient plasma for more than 100 days after HEV clearance. Similarly, prolonged fecal shedding has been shown in patients on RBV therapy, despite undetectable viremia $[21,31,32]$.

The apparent effect of RBV therapy on HEV infection depends on the viral marker of interest and the compartment analyzed. Our data indicates that in plasma RBV especially has an effect on the secretion of infectious viral particles (HEV RNA) and less on secreted ORF2 that is not associated with RNA. The differential effect on plasma versus fecal RNA suggests that when RBV interferes with viral replication, the available viral RNA is preferentially packaged into particles that are secreted into the bile-canalicular pathway rather than those secreted into plasma. Our results are in agreement with recent data published by Capelli et al., who reported that infectious HEV particles are mainly released to the bile, while only small fractions are released to the blood[33]. Importantly, HEV Ag levels increased after therapy cessation indicating that HEV Ag can be also used as a surrogate marker for HEV relapse.

Finally, we assessed the in vivo stability of HEV ORF2 in absence of HEV replication, i.e. after injection in non-humanized mice. HEV ORF2 gradually disappeared within 1 week from both feces and plasma, while viral RNA immediately became undetectable. This is faster than what we observed in our RBV treatment study, indicating that during treatment there was remaining low-level viral replication and protein secretion into the plasma. Hence also explaining the presence of HEV RNA and $\mathrm{Ag}$ in the mouse stool and relapse after therapy cessation. Although the study by Behrendt et al. 
did not mention any stool data, the persistence of HEV Ag in the patient plasma after RBV therapy must have been due to same reason. Further studies are needed to ascertain this point.

In conclusion, our results show the kinetics of HEV Ag during the course of HEV infection, therapy and relapse. The differential impact of RBV therapy on viral RNA and antigen depending on the samples type (feces vs. plasma) is important for the interpretation of HEV diagnosis and evaluation of anti-HEV therapy, especially for laboratories where molecular diagnosis is not available and HEV $\mathrm{Ag}$ is the only diagnostic marker available. 


\section{References}

1. Sayed IM, Vercouter AS, Abdelwahab SF, Vercauteren K, Meuleman P. Is hepatitis E virus an emerging problem in industrialized countries? Hepatology (Baltimore, Md) 2015; 62:1883-92. 2. Smith DB, Simmonds $P$, Jameel $S$, et al. Consensus proposals for classification of the family Hepeviridae. The Journal of general virology 2015; 96:1191-2.

3. Smith DB, Simmonds P, Izopet J, et al. Proposed reference sequences for hepatitis E virus subtypes. The Journal of general virology 2016; 97:537-42.

4. Sayed IM, Vercauteren K, Abdelwahab SF, Meuleman P. The emergence of hepatitis E virus in Europe. Future Virology 2015; 10:763-78.

5. Takahashi M, Nishizawa T, Sato H, et al. Analysis of the full-length genome of a hepatitis $\mathrm{E}$ virus isolate obtained from a wild boar in Japan that is classifiable into a novel genotype. The Journal of general virology 2011; 92:902-8.

6. Woo PC, Lau SK, Teng JL, et al. New hepatitis E virus genotype in camels, the Middle East. Emerging infectious diseases 2014; 20:1044-8.

7. Lee GH, Tan BH, Teo EC, et al. Chronic Infection With Camelid Hepatitis E Virus in a Liver Transplant Recipient Who Regularly Consumes Camel Meat and Milk. Gastroenterology 2016; 150:355-7.e3.

8. Woo PC, Lau SK, Teng JL, et al. New Hepatitis E Virus Genotype in Bactrian Camels, Xinjiang, China, 2013. Emerging infectious diseases 2016; 22:2219-21.

9. Kamar N, Selves J, Mansuy JM, et al. Hepatitis E virus and chronic hepatitis in organ-transplant recipients. The New England journal of medicine 2008; 358:811-7.

10. Wedemeyer H, Pischke S, Manns MP. Pathogenesis and treatment of hepatitis e virus infection. Gastroenterology 2012; 142:1388-97.e1.

11. Kamar N, Izopet J, Tripon S, et al. Ribavirin for chronic hepatitis E virus infection in transplant recipients. The New England journal of medicine 2014; 370:1111-20. 
12. Vollmer T, Knabbe C, Dreier J. Comparison of real-time PCR and antigen assays for detection of hepatitis E virus in blood donors. Journal of clinical microbiology 2014; 52:2150-6.

13. Gupta E, Pandey P, Pandey S, Sharma MK, Sarin SK. Role of hepatitis E virus antigen in confirming active viral replication in patients with acute viral hepatitis E infection. Journal of clinical virology : the official publication of the Pan American Society for Clinical Virology 2013; 58:374-7.

14. Tremeaux $\mathrm{P}$, Lhomme $\mathrm{S}$, Chapuy-Regaud $\mathrm{S}$, et al. Performance of an antigen assay for diagnosing acute hepatitis E virus genotype 3 infection. Journal of clinical virology : the official publication of the Pan American Society for Clinical Virology 2016; 79:1-5.

15. Zhao C, Geng Y, Harrison TJ, Huang W, Song A, Wang Y. Evaluation of an antigen-capture EIA for the diagnosis of hepatitis E virus infection. Journal of viral hepatitis $2015 ; 22: 957-63$.

16. Sayed IM, Vercouter AS, Meuleman P. Hepatitis E virus in acute liver failure: An unusual suspect? Hepatology (Baltimore, Md) 2016; 64:1837-9.

17. Behrendt P, Bremer B, Todt D, et al. Hepatitis E Virus (HEV) ORF2 Antigen Levels Differentiate Between Acute and Chronic HEV Infection. The Journal of infectious diseases 2016; 214:361-8.

18. Marion O, Capelli N, Lhomme S, et al. Hepatitis E virus genotype 3 and capsid protein in the blood and urine of immunocompromised patients. The Journal of infection 2019.

19. Montpellier C, Wychowski C, Sayed IM, et al. Hepatitis E Virus Lifecycle and Identification of 3 Forms of the ORF2 Capsid Protein. Gastroenterology 2018; 154:211-23.e8.

20. Yin $X$, Ying $D$, Lhomme $S$, et al. Origin, antigenicity, and function of a secreted form of ORF2 in hepatitis E virus infection. Proceedings of the National Academy of Sciences of the United States of America 2018; 115:4773-8.

21. Sayed IM, Verhoye L, Cocquerel L, et al. Study of hepatitis E virus infection of genotype 1 and 3 in mice with humanised liver. Gut 2017; 66:920-9.

22. Allweiss L, Gass S, Giersch K, et al. Human liver chimeric mice as a new model of chronic hepatitis E virus infection and preclinical drug evaluation. Journal of hepatology 2016; 64:1033-40. 
23. Sayed IM, Foquet L, Verhoye L, et al. Transmission of hepatitis E virus infection to human-liver chimeric FRG mice using patient plasma. Antiviral research 2017; 141:150-4.

24. van de Garde MD, Pas SD, van der Net G, et al. Hepatitis E Virus (HEV) Genotype 3 Infection of Human Liver Chimeric Mice as a Model for Chronic HEV Infection. Journal of virology 2016; 90:4394401.

25. Sayed IM, Meuleman P. Murine Tissues of Human Liver Chimeric Mice Are Not Susceptible to Hepatitis E Virus Genotypes 1 and 3. The Journal of infectious diseases 2017; 216:919-20.

26. Todt D, Moeller N, Praditya D, et al. The natural compound silvestrol inhibits hepatitis $E$ virus (HEV) replication in vitro and in vivo. Antiviral research 2018; 157:151-8.

27. Meuleman P, Libbrecht L, De Vos R, et al. Morphological and biochemical characterization of a human liver in a uPA-SCID mouse chimera. Hepatology (Baltimore, Md) 2005; 41:847-56.

28. Zhang F, Li X, Li Z, et al. Detection of HEV antigen as a novel marker for the diagnosis of hepatitis E. Journal of medical virology 2006; 78:1441-8.

29. Majumdar M, Singh MP, Pujhari SK, Bhatia D, Chawla Y, Ratho RK. Hepatitis E virus antigen detection as an early diagnostic marker: report from India. Journal of medical virology 2013; 85:8237.

30. Geng $Y$, Zhao C, Huang W, et al. Detection and assessment of infectivity of hepatitis $E$ virus in urine. Journal of hepatology 2016; 64:37-43.

31. Abravanel F, Lhomme S, Rostaing L, Kamar N, Izopet J. Protracted fecal shedding of HEV during ribavirin therapy predicts treatment relapse. Clinical infectious diseases : an official publication of the Infectious Diseases Society of America 2015; 60:96-9.

32. Ambrosioni J, Mamin A, Hadengue A, et al. Long-term hepatitis E viral load kinetics in an immunocompromised patient treated with ribavirin. Clinical microbiology and infection : the official publication of the European Society of Clinical Microbiology and Infectious Diseases 2014; 20:071820. 
33. Capelli N, Marion O, Dubois M, et al. Vectorial Release of Hepatitis E Virus in Polarized Human Hepatocytes. Journal of virology 2018. 
Author contributions: IMS, CM, LC and PM designed the experiments. IMS, LV and CM performed experiments. IMS, LC, , CM and PM analyzed data. FA and JI provided essential reagents. IMS and PM wrote the manuscript. PM conceived and supervised the study.

Funding This study was funded by the Ghent University and the Lille 2 University (IRO project MODEL-HEPE), The Research Foundation-Flanders (FWO-Vlaanderen; projects GOD2715N, G047417N and EOS project VirEOS30981113), the Agency for Innovation by Science and Technology (IWT SBO project HLIM-3D), and the 'AgenceNationale de Recherches sur le Sida et les hépatitesvirales' (ANRS). IMS is a recipient of a PhD fellowship provided by the Egyptian Government and Ghent University.

Competing interests None. 
Figure 5: Kinetics of HEV Ag during ribavirin therapy in humanized mice.

Humanized mice $(n=5)$ were treated orally with RBV for 2 weeks. HEV Ag $(A)$ and HEV RNA (B) were measured at start (SOT) and end of therapy (EOT). HEV Ag was monitored in two humanized after RBV therapy cessation (C). Fecal and plasma data are shown in black and red respectively. Horizontal lines represent the geometric mean. LOQ: is limit of quantification. 
Figure 1
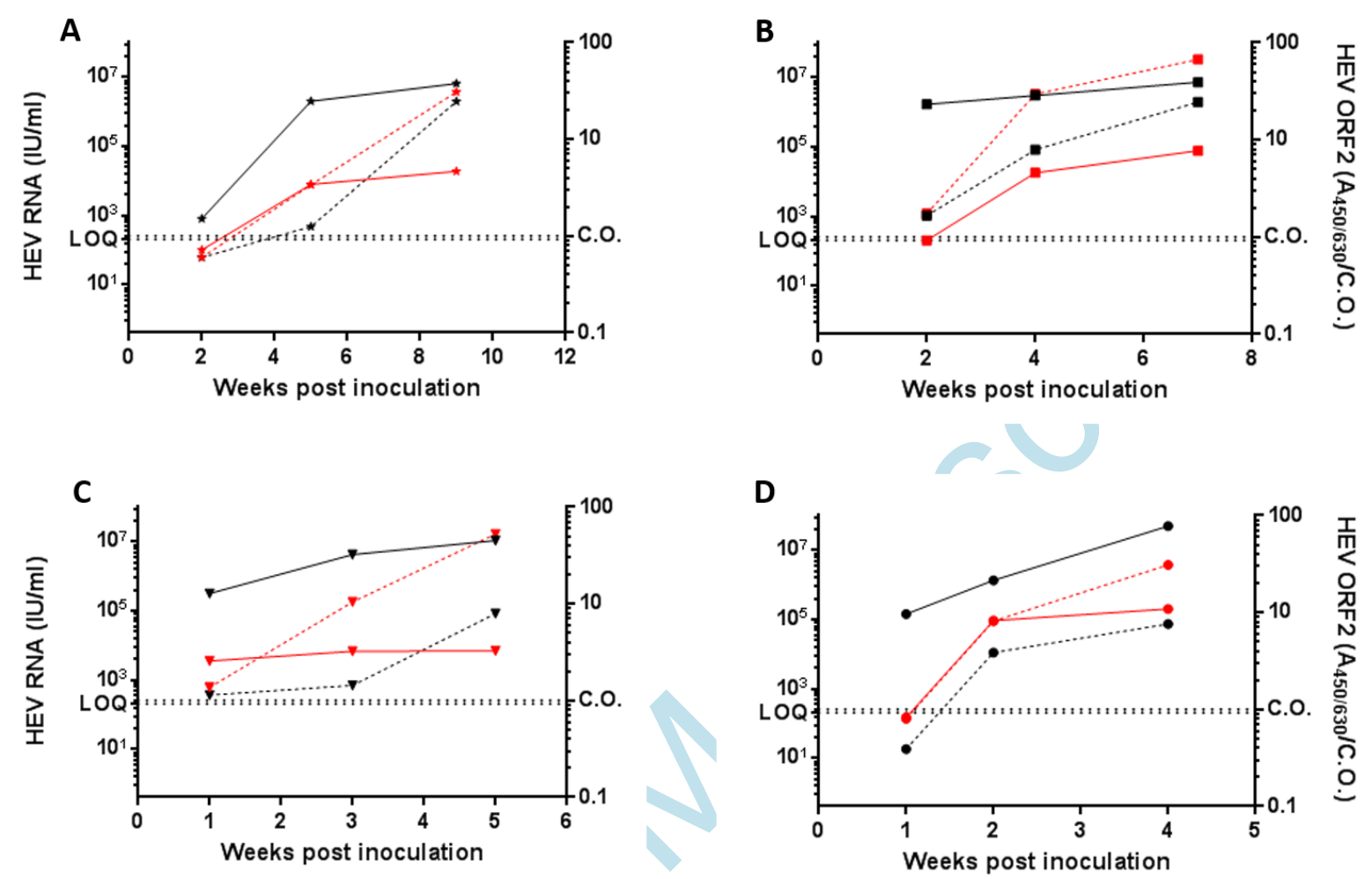
Figure 2
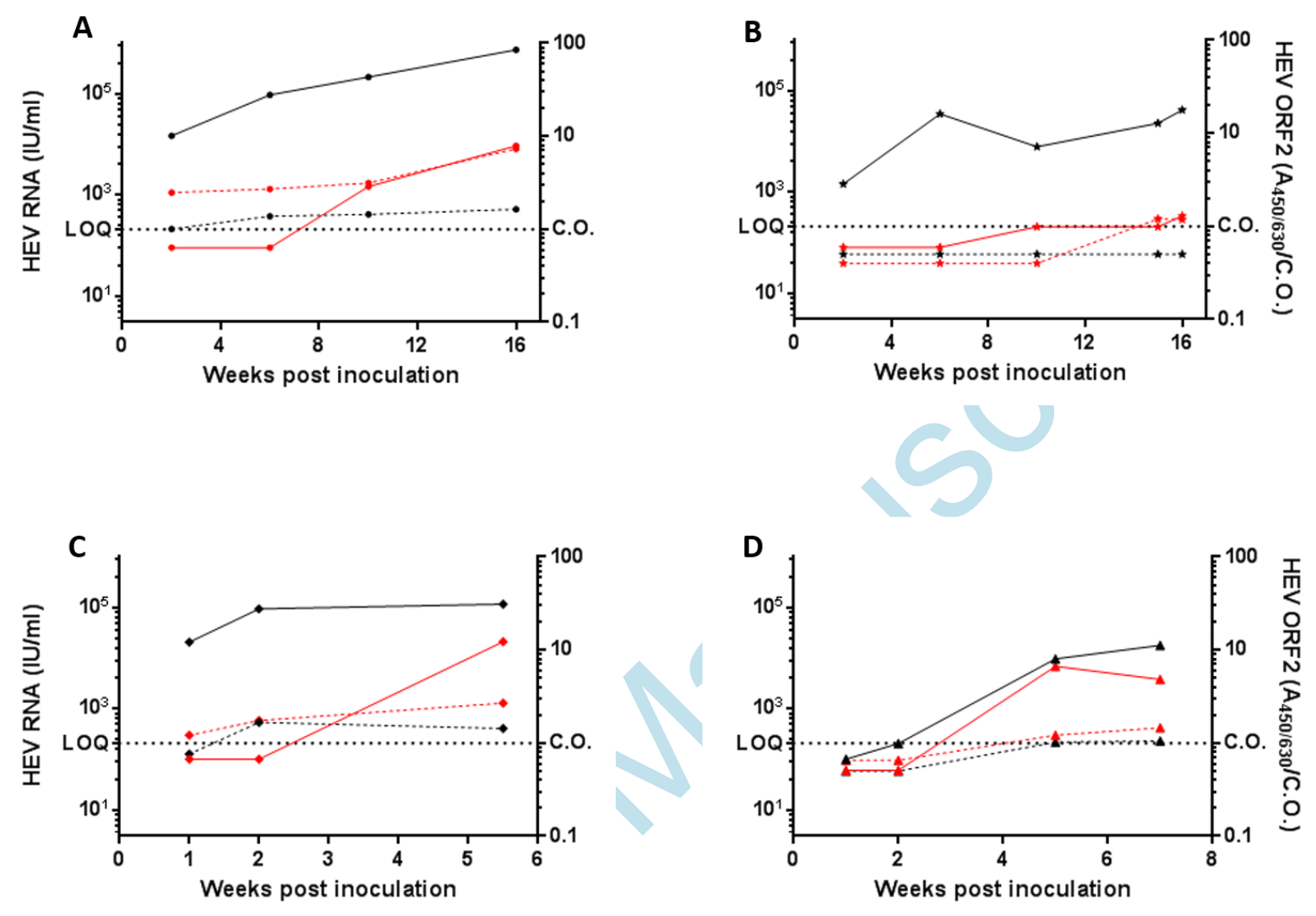
Figure 3
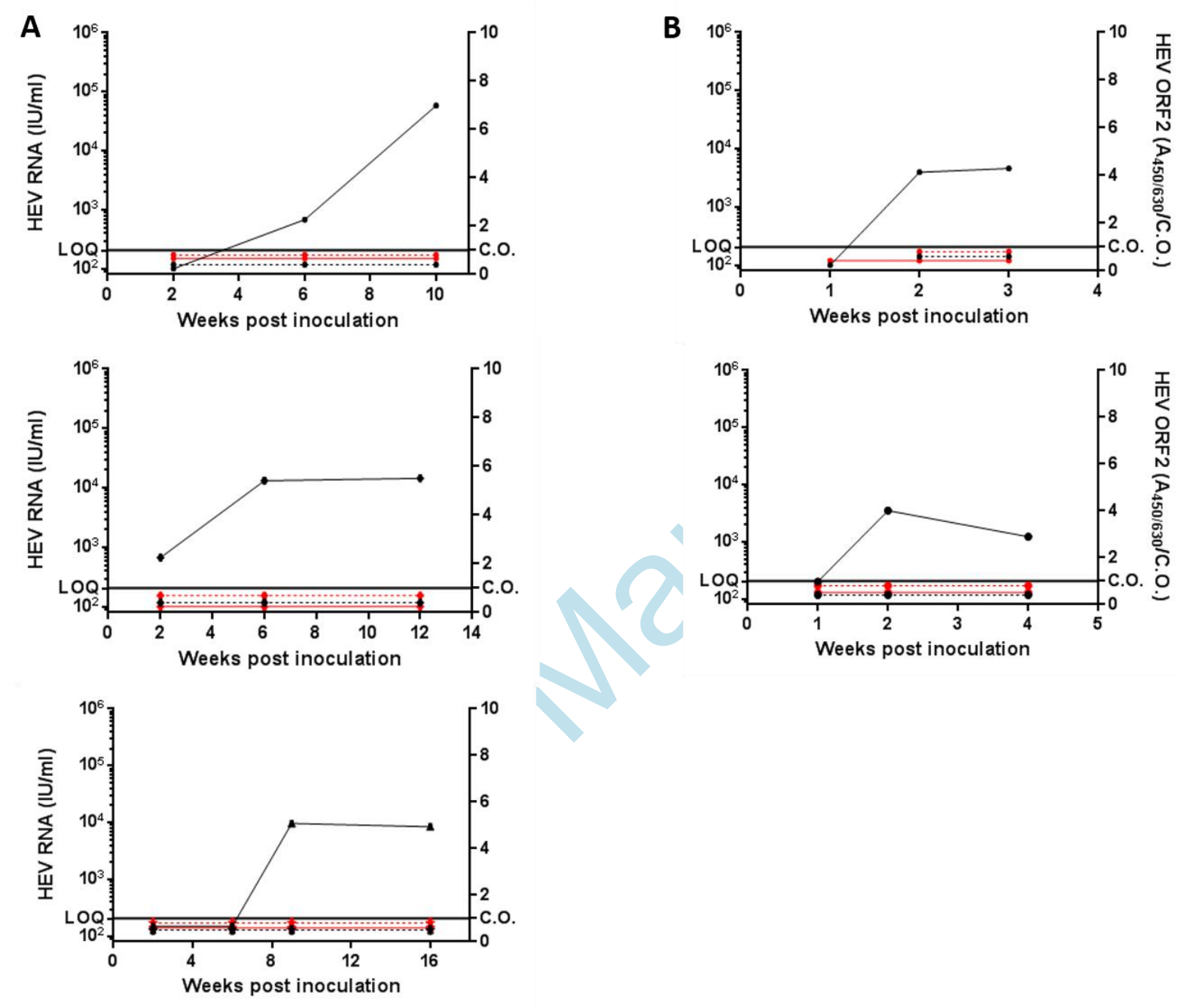
Figure 4
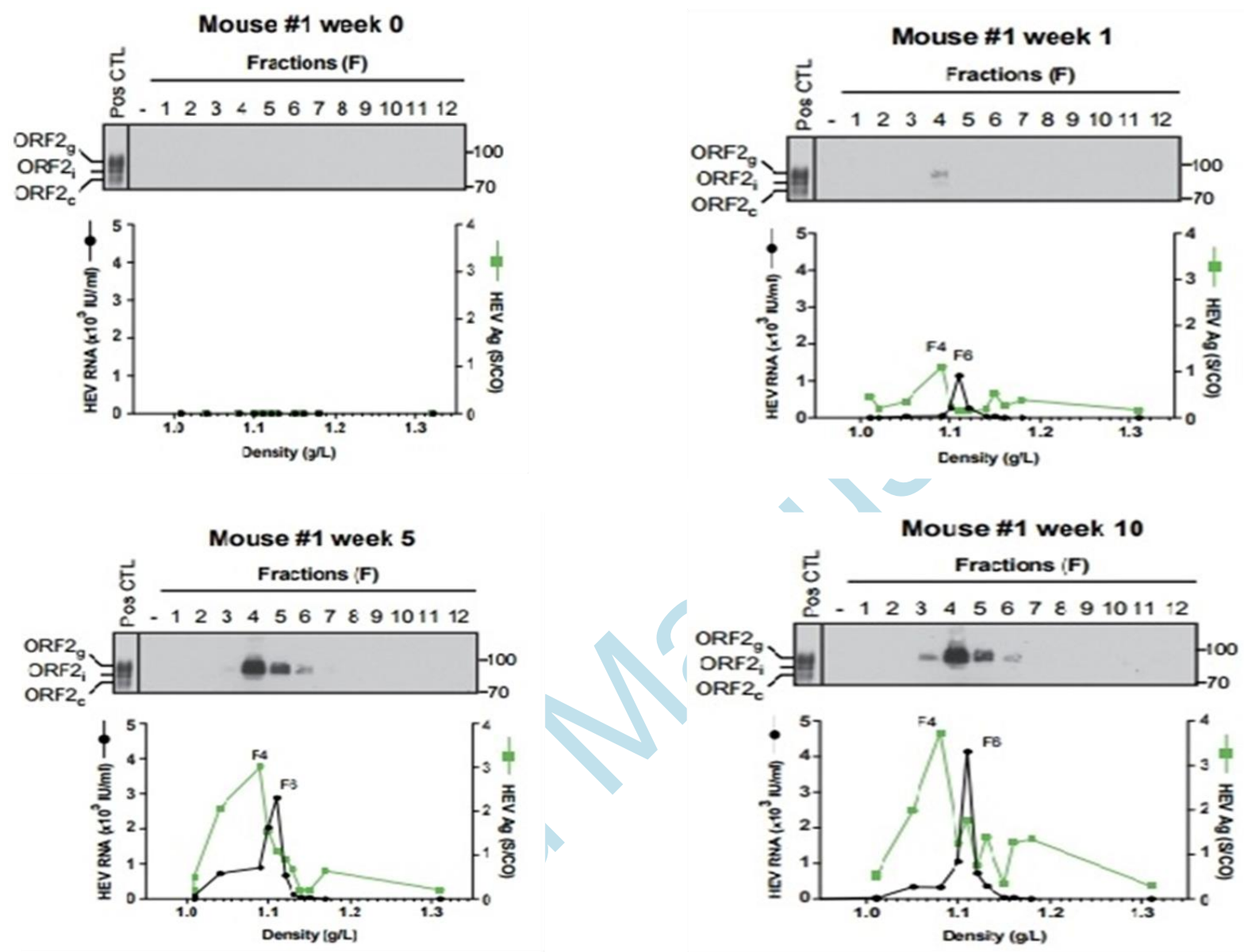
Figure 5
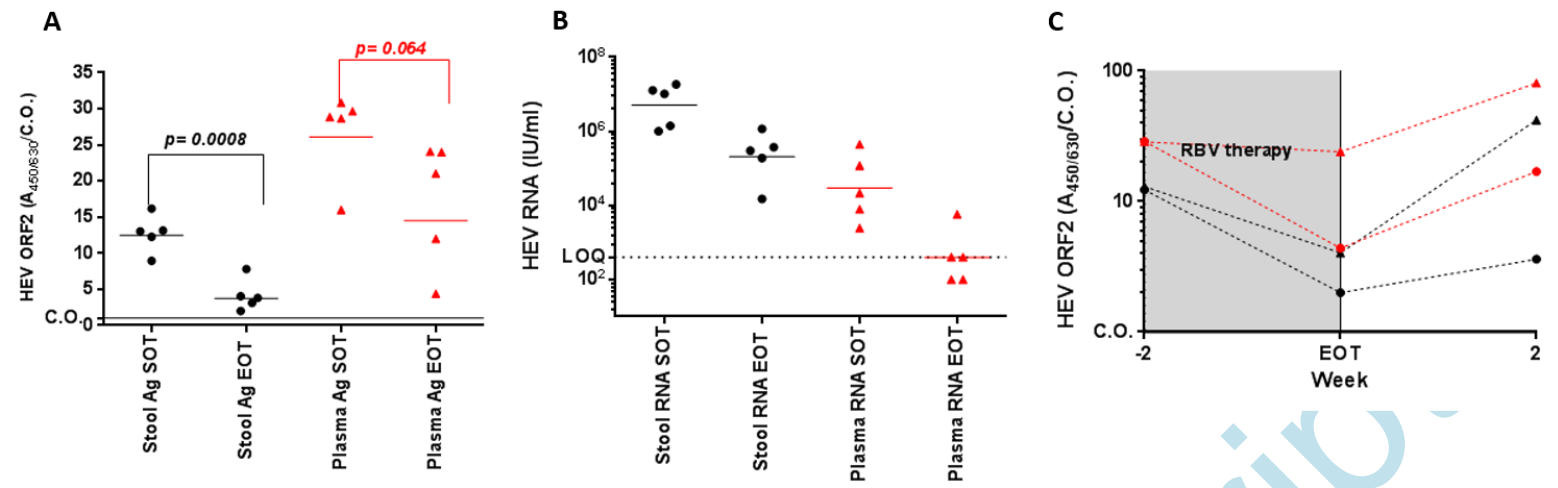